\title{
Fundamental Physics used in Forensics
}

\author{
Mukesh Sharma* \\ Assistant Director, India
}

ISSN: 2578-0042

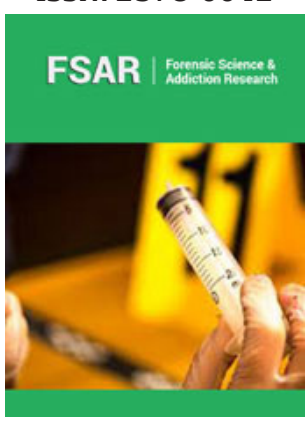

*Corresponding author: Mukesh Sharma, Assistant Director (Physics Division), Regional FSL, India

Submission: 留 June 12, 2019

Published: 侮June 28, 2019

Volume 5 - Issue 1

How to cite this article: Mukesh Sharma. Fundamental Physics used in Forensics. Forensic Sci Add Res. 5(1). FSAR.000606.2019.

DOI: 10.31031/FSAR.2019.05.000606

Copyright@ Mukesh Sharma, This article is distributed under the terms of the Creative Commons Attribution 4.0 International License, which permits unrestricted use and redistribution provided that the original author and source are credited.

\begin{abstract}
Because of great number human activity that a human might be involved in a crime or at a crime scene, the quantity of physical material that could be use as physical evidence is almost non detectable with human eye. These evidences may be microscopic in character, such as DNA or a tiny particle of human body like skin, semen or hair and sometime quite like a motor vehicle left behind at the scene, weapon at spot of occurrence etc. Forensic evidence and physical evidence provide most of the era in which forensic expert (Physics), also known as scientists, can explain his/her expertise with using fundamental physics. Directly fundamental physics having a great contribution in the field of forensic science via ray optics, laser sources and modern physics and many more. Instrumentation like optical microscope, the electron microscope, the mass spectrometer, and optical spectrometers (optical physics); but directly, physical sciences role in forensic science has been used very few scientists due lack of education. Forensic physics in crime labs have been traditionally involved the measurement of density (soil and glass examination), index of refraction, and birefringence (fiber analysis, glass examination), restoration of VIN, Building material analysis etc. In the 3 decades, the use of the photoluminescence, EDX, XRF, SEM and XRD phenomenon for physical evidence examination has emerged material characterization, with latent fingerprint detection the most notable application. Last one decade the analog and digital electronics (Cyber Crime) has been dominating the field of forensic science. The author has tried to stream Physical Sciences in forensic sciences is summarized here for the new generation those are willing to work in the field of forensic sciences. The minimum and eligibility criteria for a forensic person in India are also explained in the article.
\end{abstract}

\section{Introduction}

Forensic sciences deal with the application of scientific knowledge to legal problems and are vital tools for unearthing the truth in any legal proceeding. In some subareas of forensic science, for example, physical sciences and various engineering disciplines, including mechanical, electrical, and chemical engineering, and fire science, are also involved in forensic investigations, often in civil cases, but also in criminal investigations. Crime scene expert must be able to apply the techniques of the physical sciences and biological sciences, while examining evidence at scene of occurrence or in lab, with the goal to prove the existence of a crime scene and to connect suspects to the crime in the court of law. The evidences the forensic scientist collects at scene of occurrence handed over to investigating authority should be submitted with proper way means maintaining chain of custody $[1,2]$ in the court of law. In the field of forensic science is dominated by the fields of chemistry (mainly drug and toxicology) and biology (serology and DNA profiling). But due to the wide range of physical evidence, the forensic experts often specialize in a particular area with master or doctoral degree. But this all, no matter what the specialty an expert has, he/she must be able to examine, analyze, identify, and interpret physical evidence collected from the scene of occurrence.

Reconstruction of crime scene is a vital application of Physics in forensic science as the cases are belongs to accidents, suicide or homicide, fall of a body from height, projectile motion of a firearm, glass penetration of speedy stone, bullet or tool case be analyzed using physics [3]. The Forensic scientists examine thoroughly the physical evidence collected from the crime scenes to help to establish the nature of a crime with the criminal. It is an general perception that much of analytical part of forensic sciences are dependent upon chemistry and the analysis of chemical compositions. But very few scientists is agreed and known that how the forensic scientist makes use of physical sciences in his regular investigation of evidences [4]. 


\section{Physics division and its classification dealing the forensic science in India}

A recent innovation in some physics departments is the introduction of courses on forensic physics. In the Physical sciences for forensic person the topics of interest include road accidents analysis, firearm and bullets profile analysis, fire, arson and explosion investigation, materials identification (fake jewellery etc.) and software-based imaging methods for facial reconstruction etc [1-5]. Another topic that could be used to illustrate a forensic application of Newton's laws, momentum conservations laws and blood pattern analysis using trigonometry and the physics of falls from a height involving serious injury or death. These all, motivates to an educator to work on the Physics with new forensic applications $[5,6]$. In India, Physics Division in State FSLs and Central FSLs mainly deals with all types of cases, beginning from reconstruction of crime scene, trace evidences analysis to voice examinations. One can easily determine the classification of Physical sciences branches in forensic sciences those who are working in the field of forensic science, but those are aspirant to develop their carrier in the field of forensic sciences must go through this article, it will help him/her to plan their carrier in the Physics within forensic science. Mainly physics can be categories in the five fields as shown in the Figure 1. These categories are General Physics (General and Forensic Engg. Physics), Reconstruction of CS, Forensic Accident Analysis, Material Science, Analog and Digital Electronics.

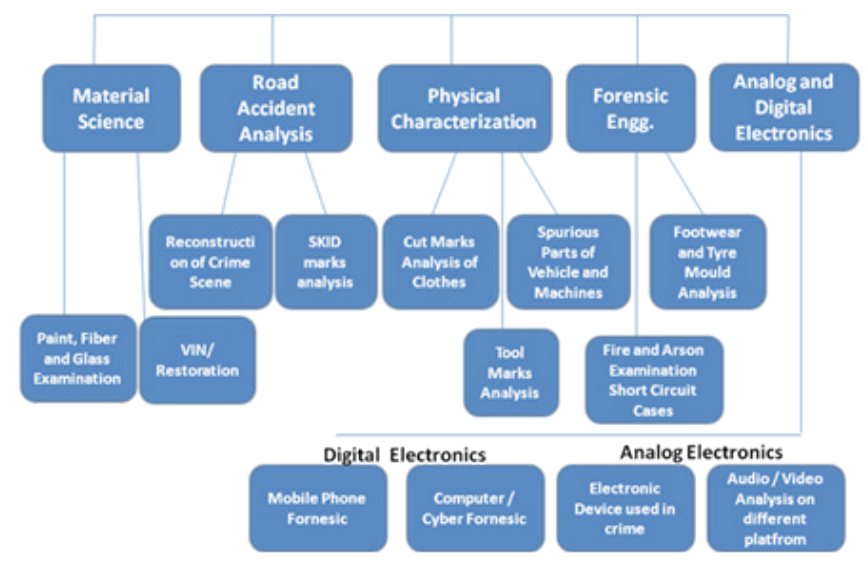

Figure 1: Branches of forensic science those are dealt by physics divisions in India.

\section{General physics}

Starting with the laws of motion which generally covered in engineering studies and as well in courses in physics, dynamics, or kinematics as shown in Figure 2 \& 3. General Physics in forensic science involves electrical, mechanical, chemical, and laboratory analysis as well as mathematical formulations based on recognized principles of fundamental physics. The fundamental physics like conservation law of momentum, collision, Newton's law of motions are having a great and vital application in reconstruction of scene of crime. All the fundamental laws of Physics are very much helpful to analyze the evidence as well and draw any conclusion.

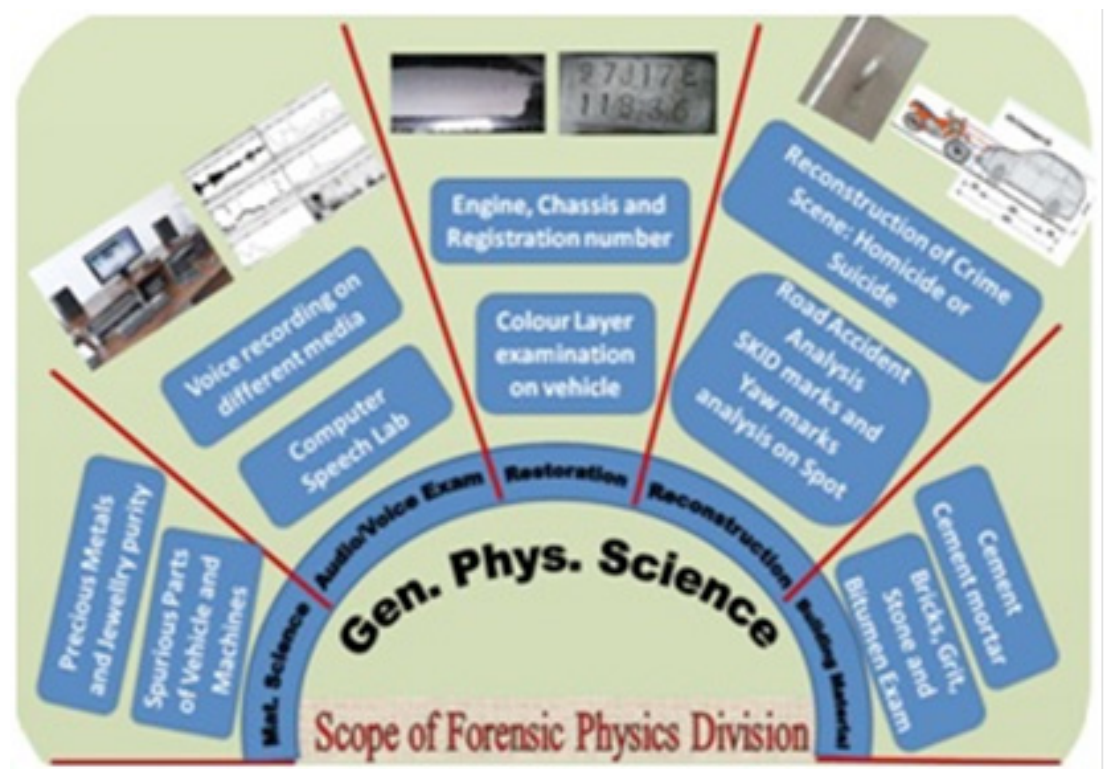

Figure 2: Showing the gen physics section deal in physics division of state FSLs and CFSLs. 


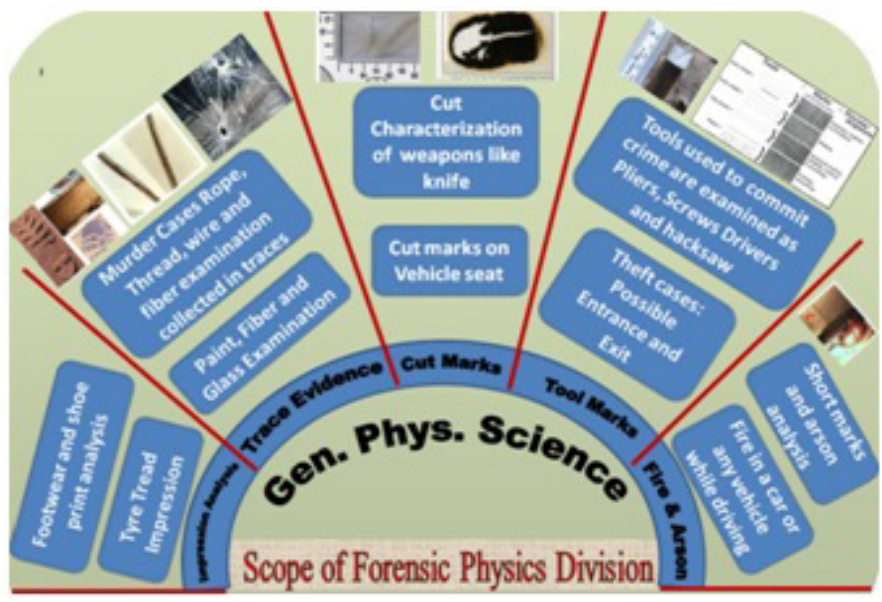

Figure 3: Showing some more gen physics section deal in physics division of state FSLs and CFSLs in India.

\section{Forensic engineering and building material analysis}

Forensic engineers and building material lab analyze why things fail. In physical science and engineering while manufacturing faulty toaster that electrocute people, to buildings, over bridges and river bridges that collapse, and many people died due the incident of faulty construction or faulty manufacturing. The best example for the forensic engineering is the September 11 attacks on the world trade center and the Pentagon, which assisted greatly to the forensic science in the analysis and reconstructed the scene of occurrence. Forensic engineers is also useful to reconstruct road accidents, electric fire etc. On the basis of tire skid marks, damage on the collided vehicles and surrounding scenario and physical evidences by using the law of physics forensic expert may easily determine path, direction, speed, and the type of collision that occurred or what might happen (Figure 4).

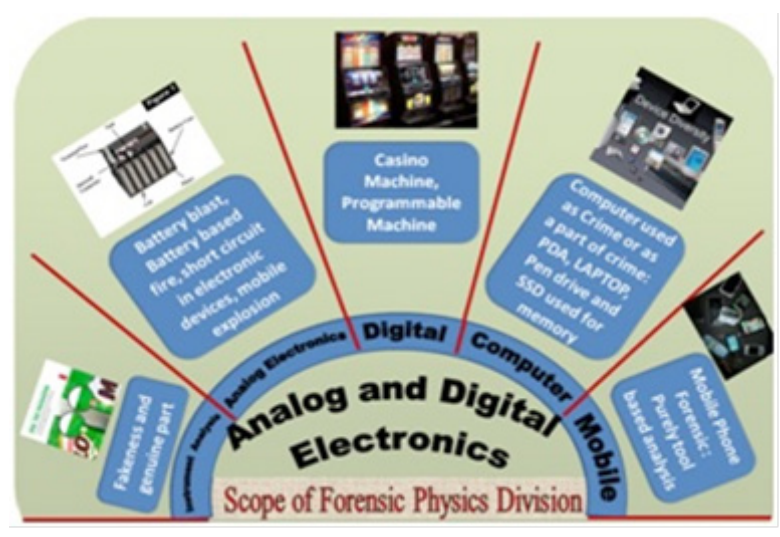

Figure 4: Showing analog and digital electronics having forensic application.

\section{Specialized reconstruction of cs and accident analysis}

Reconstruction of crime scene involves train accidents, suicides/ homicides; accidents reconstructions and dead body when fall from height. On the grounds of skid marks analysis, yaw marks analysis, break pattern and other parameters observed at road accident help to reconstruct the scene of occurrence. Similarly, in dead body fall from height may be calculated on basis of fundamental physics.

\section{Material science}

Materials science is a relatively new discipline which emerged in the 1960's to provide a comprehensive view and approach to the study of materials using solid state physics, quantum chemistry and metallurgy. It has a wide range of application forensic sciences too using interaction of radiation with matter like X-rays and Gamma-rays. Using the phenomenon of X-rays based nondestructive techniques like XRD, XRF, ED-XRF and SEM became useful instrument those are widely used in the forensic science laboratory. Recent work has shown that materials science can make a substantial and growing contribution to criminalistics. As a result, forensic materials science may become a field of its own in the future in last two decades. Material science has a vital role in analysis of the minute evidences collected from the scene of occurrences with the help of analytical techniques.

\section{Analog and digital electronics}

The electronic is purely subject of physics; analog electronic is useful for cases belong to fire and arson, sometime explosive material cases. The electronics devices used to commit crime are come is this category. Analog advance physics comes with subcategories as digital electronic in these categories, the entire computer-based crime and mobile those have internal memories are coming within this range. 


\section{Ballistic evidence}

Another important use of physical sciences in the field of the ballistic, which is purely deal with firearms, bullets and ammunition. Ballistic division are work separately in Indian forensic science laboratory at State and central level. The evidences related to ammunition or firearms can sometimes be used to determine the trajectory of a bullet and its potential impact on the item it strikes, whether it be a person or an object with help of fundamental physics. Sometime in the reconstruction of firearms incident, projectile motion and trajectory motion analysis is very useful. The Forensic scientists deal with ballistics need to understand how force and trajectory impact an object that is struck which is also an application of fundamental physics. In the firearms analysis the GSR study has a great value, which can be analyzed using established chemical testing kits or non-destructive techniques like EDX and SEM, determine the exact results. The velocity measuring instrument for gun chronograph works on the sound waves.

\section{Discussion}

The advancement in the field of forensic sciences, the application of physics is well established and growing day by day research and development. In this context forensic scientists those are working in the field which need specialized knowledge in physics to analyze the items of physical evidences into reports and testimonies in the court of law. Knowledge of analysis of the evidence is required in physics as in important and fundamental subject to be studied to the forensic students. So that, in part, sets the limits of its interpretation within the physics laws and applications. An academic base is required for forensic science to mature as a science and profession. We as forensic community should recommend that the Physics as basic subject for forensic university and forensic department to teach the student, those are non-physics background. The "formal" aspect of academic education is required, however, can run afoul of those who see the formalities as being at least as and sometimes more important than the knowledge itself. Formal education can also fall prey to overvaluing immediately useable, but 'practical' knowledge, and underrate the importance of fundamentals, and of wisdom altogether is made a proper forensic scientist.

\section{References}

1. Houck MM, Siegel JA (2010) Fundamentals of Forensic Science, $\left(2^{\text {nd }} e d n\right)$, Academic Press, San Diego, USA.

2. Lewis PR, Reynolds K, Gagg C (2003) Forensic Materials Engineering: Case Studies, CRC Press, Boca Raton, Florida, USA.

3. Noon RK (2001) Forensic Engineering Investigation, CRC Press, Boca Raton, Florida, USA.

4. Li CT (2011) Computational Forensics, Digital Crime, and Investigation Yurchak Printing Inc, Hershey, USA.

5. Franck H, Franck D (2013) Forensic Engineering Fundamentals ( $\left.1^{\text {st }} e d n\right)$, CRC Press, Boca Raton, Florida, USA.

6. Toni F (2009) Physicists in forensics. Physics Today 62(3): 20.

For possible submissions Click below: 